\title{
Jet analysis by Deterministic Annealing
}

\author{
L. Angelini ${ }^{a, b, c}$, P. De Felice ${ }^{a, b}$, M. Maggi ${ }^{c}$, G. Nardulli ${ }^{a, b, c}$, \\ L.Nitti $^{b, c, d}$, M.Pellicoro ${ }^{a, b, c}$ and S. Stramaglia ${ }^{a, b, c}$ \\ ${ }^{a}$ Dipartimento Interateneo di Fisica, Bari, Italy \\ ${ }^{b}$ TIRES, Center of Innovative Technologies for Image Detections and \\ Processing, Bari, Italy \\ ${ }^{c}$ I.N.F.N., Sezione di Bari, Italy \\ ${ }^{d}$ DETO, Università di Bari, Italy
}

\begin{abstract}
We perform a comparison of two jet clusterization algorithms. The first one is the standard Durham algorithm and the second one is a global optimization scheme, Deterministic Annealing, often used in clusterization problems, and adapted to the problem of jet identification in particle production by high energy collisions; in particular we study hadronic jets in $W W$ production by high energy $e^{+} e^{-}$scattering. Our results are as follows. First, we find that the two procedures give basically the same output as far as the particle clusterization is concerned. Second, we find that the increase of CPU time with the particle multiplicity is much faster for the Durham jet clustering algorithm in comparison with Deterministic Annealing. Since this result follows from the higher computational complexity of the Durham scheme, it should not depend on the particular process studied here and might be significant for jet physics at LHC as well.
\end{abstract}

PACS Numbers: 13.87.-a, 13.38.Be, 05.10.-a, 45.10.Db

\section{Introduction}

The problem of clustering consists of optimal grouping of observed signal samples (set of features). In many circumstances one seeks the partition of the given set of features which minimizes a prescribed cost function. This function should embody the a priori knowledge on the geometrical aspects of the problem. By this way clustering is thus transformed in an optimization task. The main applications of clustering are in pattern recognition and signal compression. Here we address a different problem, i.e. the task of 
partitioning particles produced in high energy $e^{+} e^{-}$annihilation into a certain number of cone regions, i.e. small solid angles. This is the well known problem of jet clustering in high energy physics. It arises from the need to relate energy and momentum of the cluster (jet) to the four-momentum of the underlying and unobservable parton. There are at the moment a few well established jet clustering algorithms, to be reviewed in section 2. While tuned to the particular problem at hand they were not studied from the point of view of the theory of clustering algorithms and are not generally considered as minimization problems of prescribed cost functions. We feel this is a matter to be further investigated and therefore in this letter we wish to raise a few questions: Can the existing jet algorithms result from some variational principle? Can the general theory of clustering algorithms be used to reduce the computational time of jet algorithms? In this letter we answer these questions by considering a specific algorithm, the Deterministic Annealing (DA) [1, 2], to be reviewed in section 3; using an analogy with statistical physics, DA relates the problem of clustering to that of finding the global minimum of a thermodynamical potential. In order to compare this method to the traditional ones we will study a specific issue, i.e. the production of jets in $W W$ production in high energy $e^{+} e^{-}$colliders. This application will be discussed in section 4 . Our results are contained in section 5; they show that the jet clustering algorithms and Deterministic Annealing give similar performances, but the computational complexity of DA is considerably lower; the use of methods based on variational principles, such as DA, would be therefore certainly of interest in the analysis of hadronic final states at the future accelerators such as the Large Hadron Collider at CERN, where the multiplicities might be very high and the computational complexity extremely demanding for ordinary jet clustering algorithms.

\section{Jet clustering algorithms}

The most common jet clustering algorithms used in studies of $e^{+} e^{-}$annihilation are the JADE [3], Durham [4] and Cambridge [5] algorithms. The prototype of these clustering methods and the oldest one is the JADE algorithm. One considers all the possible pairs $(i, j)$ of particles in the final state, with energies $E_{i}, E_{j}$, and angular separation $\theta_{i j}$ and computes the jet

\footnotetext{
${ }^{1}$ For a review of these and other jet algorithms see [6]. For a review of the Montecarlo generators and their connections with the jet algorithms see [7].
} 
resolution variable

$$
y_{i j}=y_{i j}^{J} \equiv \frac{2 E_{i} E_{j}\left(1-\cos \theta_{i j}\right)}{E_{v i s}^{2}},
$$

where $E_{v i s}$ is the visible energy, i.e. the sum of energies for all particles observed in the final state. This test variable is then compared to a given threshold parameter $y_{c u t}$ and the pair is recombined into a new pseudo-particle $k$ of four-momentum

$$
p_{k}=p_{i}+p_{j}
$$

( $E$ scheme) provided that $y_{i j} \leq y_{\text {cut }}$. The algorithm is then reiterated to the new set of (pseudo)particles and it stops when, for all pairs, $y_{i j} \geq$ $y_{\text {cut }}$. The number of pseudoparticles at the end of the algorithm counts the number of jets, which is therefore fixed by $y_{\text {cut }}$. The theoretical advantage of this recombination scheme lies in the absence of collinear and infrared singularities, as the regions of phase space where these divergences could be generated are automatically excluded.

A drawback of the definition (11) is that also particles at very different angles can be recombined in one pseudoparticle. This is due to the fact that $2 E_{i} E_{j}\left(1-\cos \theta_{i j}\right) \approx M_{i j}^{2}$, where $M_{i j}^{2}$ is the invariant mass of the two particles. On the theoretical side this implies the presence of large order corrections that cannot be resummed; on the experimental side the trouble is that ghost jets may appear i.e. jets along directions where no particles are present. To cure this problem the Durham algorithm was introduced, which is based on the following definition of the test variable:

$$
y_{i j}=y_{i j}^{D} \equiv \frac{2 \min \left\{E_{i}^{2}, E_{j}^{2}\right\}\left(1-\cos \theta_{i j}\right)}{E_{v i s}^{2}} .
$$

Clearly the resolution criterion $y_{i j}^{D}>y_{c u t}$ becomes, for small angles, $k_{T i}^{2}>$ $E_{v i s}^{2} y_{\text {cut }}$, where $k_{T i}$ is the transverse momentum of the $i$-th particle to the direction of the $j$-th one. In this way the algorithm tries to minimize the transverse momentum and not the invariant mass. On the other hand the recombination scheme is still given by (2).

The Durham algorithm presents a problem at very small values of $y_{\text {cut }}$. As a matter of fact, when one tries to resolve the final state to get a larger number of jets, particles that are almost collinear can be recombined, thus producing unwanted junk jets. This feature is solved by the Cambridge algorithm by introducing a third step in the process of formation of the clusters. Before 
considering the jet resolution and the recombination steps, one introduces an ordering variable $v_{i j}=2\left(1-\cos \theta_{i j}\right)$. Once the pair $(k, \ell)$ with the minimum value of $v_{i j}$ is found, the resolution variable $y_{k \ell}$ (still given by (3)) is computed and, after a comparison with $y_{\text {cut }}$, the recombination scheme (2) is applied?

In this paper we are not interested in the analysis of small $y_{\text {cut }}$ as we consider the production of a $W$ pair in $e^{+} e^{-}$scattering, i.e. a fixed (four) number of jets. In this context the Durham and the Cambridge algorithms produce analogous results. Therefore in the sequel we will mainly refer to the Durham algorithm. For a more detailed comparison among jet algorithms one can however see 8$]$.

\section{Deterministic annealing}

Deterministic annealing algorithms [1] (for reviews see [2]) take their name from the annealing procedure in physical chemistry. This process starts from a metastable state of the metal, reached by a sudden decrease of temperature; the annealing procedure consists in a gradual cooling by which the mineral passes from the metastable phase to the low temperature minimum of energy. During the annealing procedure the system passes through states of thermal equilibrium and, correspondingly, of minimal Helmholtz free energy $F$. In the limit of low temperatures one is therefore guaranteed that the system is in the global minimum and not in a local minimum of $F$.

A computational method trying to simulate annealing was invented about twenty years ago [9], based on the Metropolis algorithm 10. This method, called Simulated Annealing (SA) not only simulates annealing in its quest for the global minimum of the free energy, but also in its stochastic evolution; because of this last feature it can become rather time-consuming. This snag is avoided in Deterministic Annealing, the method we will use here. It is still an annealing method because it points to the global minimum of $F$ and allows gradual cooling of the system, but it is deterministic since the procedure of optimization (see below) is obtained deterministically and not by a random process. In the sequel we shall describe DA in general terms, while in the next section we shall discuss the modifications we have implemented to adapt it to the particular problem of the determination of the four jets in $W W$ production in $e^{+} e^{-}$diffusion.

\footnotetext{
${ }^{2}$ The Cambridge algorithm implements the so called soft freezing, i.e. if the particles are not recombined the softer particle is removed and considered as a resolved jet.
} 
To start with, one defines two sets, the set of the data points $x \in X$ and the set of the representative points $y \in Y$, also called code - vectors, i.e. the points that eventually represent the clusters. One also defines a distance $d(x, y)$ between the data point $x$ and the code-vector $y$. In jet physics $x \equiv p^{\mu}$, i.e. the four momentum of one of the particles in the final state, while $y$ is the four momentum of a cluster; as for the distance $d\left(x_{i}, x_{j}\right)$ we will take it coincident with $y_{i j}$, i.e. one of the basic distances in the jet physics.

DA fixes the code-vectors by minimizing the Helmholtz free energy

$$
F^{*}=-T \sum_{x} \ln \left(\sum_{y} e^{-d(x, y) / T}\right)
$$

with respect to the code vector. Basic ingredient of the calculation is the use of $F^{*}$ as free energy; it is based on the use of the conditional probabilities

$$
p(y \mid x)=\frac{e^{-d(x, y) / T}}{Z_{x}}
$$

with

$$
Z_{x}=\sum_{y} e^{-d(x, y) / T} .
$$

It is well known that the Gibbs distribution (5) is the minimum point of the free energy, defined in general as

$$
F=D-T H .
$$

Here the role of the energy is played by

$$
D=\sum_{x, y} p(x, y) d(x, y),
$$

where $p(x, y)$ is the joint probability of $x$ and $y$ and the entropy is the Shannon entropy, i.e.

$$
H=-\sum_{x, y} p(x, y) \ln p(x, y) .
$$

By minimizing $F$ in (7) one gets $F^{*}$ provided the Gibbs distribution is used; therefore the use of the probability (5), together with the algorithmic search for a minimum of $F^{*}$ in the variables $y$, is tantamount to the quest of the global minimum of the Helmholtz free energy. 
The optimal code-vectors $y$ are obtained by solving the equations

$$
\sum_{x} p(y \mid x) \nabla_{y} d(x, y)=0
$$

which is found by the above mentioned procedure of minimization of $F^{*}$.

During the cooling process $(T \rightarrow 0)$ one encounters phase transitions that are signalled by an increase of the number of clusters. This shows the similarity between DA and the jet clustering algorithms, where the number of clusters can also increase by a reduction of the parameter $y_{\text {cut }}$. This aspect is not particularly relevant here, since the number of jets is held fixed. Nevertheless we discuss it for two reasons. First, it allows to stress an important aspect of DA that we will modify in section 5 for the application in jet physics, i.e. the definition of the optimal code-vectors; second, it could be useful in other applications where the number of jets is not fixed a priori.

To increase the number of clusters one starts with a high value of $T$; it can be shown that in the limit of very high temperatures the minimum condition (10) has one degenerate solution (all the $y$ equal). This corresponds to one cluster and, in the statistical mechanics analogy, to a completely disordered phase, typical of a high temperature state. After computation of this unique value $y_{1}$ by $(10)$, one performs a deterministic updating according to the formula

$$
y_{1}=\sum_{x} x p(x) p\left(y_{1} \mid x\right)
$$

If a pre-chosen convergence test is satisfied, one decreases the temperature. One can show that there are phase transitions when a value $y_{0}$ is found such that

$$
\operatorname{det}\left[1-\frac{2}{T} C_{x \mid y_{0}}\right]=0
$$

where $C_{x \mid y}$ is the covariance matrix computed with the (posterior) conditional probability $p(x \mid y)$. If a solution of (12) is obtained for a certain critical temperature

$$
T=T^{1 \rightarrow 2},
$$

then the previous set of code-vectors corresponds no longer to a minimum of the free energy. Therefore one adds a new cluster (therefore there are now two code-vectors, $\left.y_{1}, y_{2}\right)$ and the procedure of optimization is repeated. In general, instead of (11) one has

$$
y_{j}=\frac{\sum_{x} x p(x) p\left(y_{j} \mid x\right)}{p\left(y_{j}\right)} .
$$


The algorithm continues until a pre-fixed temperature (or number of clusters) is reached. It is worth noticing that this procedure does not assign uniquely each data point to a cluster, because some points can remain unassigned [1]. Therefore, in the final step, one cools down the system $(T \rightarrow 0)$ until all the particles are assigned.

\section{Deterministic annealing and $\mathrm{W}$ masses in $e^{+} e^{-}$diffusion}

A $W$ pair created in $e^{+} e^{-}$annihilation produces in the subsequent evolution four jets; the study of this multi-jet final state is one of the methods for the determination of the $W$ mass since the four jets can be divided into two pairs, each having as invariant mass $m_{W}^{2}$. The world average for this parameter is 11

$$
m_{W}=(80.422 \pm 0.047) \mathrm{GeV} / \mathrm{c}^{2},
$$

while the measurement at LEP, obtained combining both the hadronic and semileptonic channels, is [12]

$$
m_{W}=(80.450 \pm 0.039) \mathrm{GeV} / \mathrm{c}^{2} \text {. }
$$

Given the exploratory character of this paper we are less interested in the prediction of the actual experimental data than in the comparison of the DA and jet algorithms; therefore we choose to work with Montecarlo generated data. The data set consists of about 1500 events produced at the LEP energies by the KORALW generator [13], which includes all the four-fermion diagrams contributing to $W^{+} W^{-}$-like final states. It produces the primary reference sample, with a $W$ mass of $m_{W}=80.35 \mathrm{GeV}$ (Fig.11). The KORALW generator is interfaced with JETSET [14 for fragmentation.

There is one modification to be implemented in the DA algorithm for its use in the present study. As we have mentioned above, by eq. (14) one would extract the code-vectors. Eq. (14) would produce in the present case a vector representative of the jet corresponding to the average 4-momentum in the jet $J$; however in all the jet clustering algorithms discussed in section 2 the momentum of the single particle is compared to the total momentum of the jet $J$ and not to the average momentum of the particles in the cluster $J$. In order to reproduce this feature we modify the DA algorithm by imposing only minimization in the probability distribution $p\left(y_{j} \mid x\right)$ and not also in 


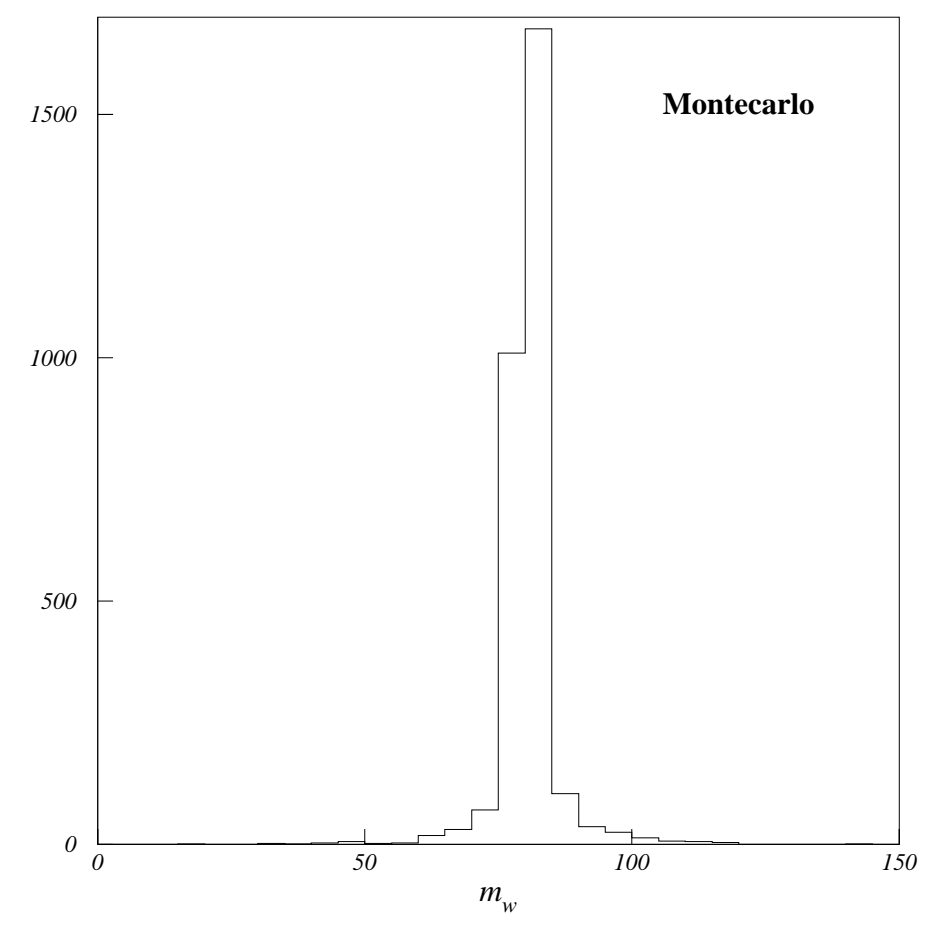

Figure 1: The $W$-mass distribution of the actual data; $m_{W}$ in $\mathrm{GeV} / \mathrm{c}^{2}$.

the variables $y$. In practice we use Eqns. (因9), but not (10) and (14); in particular we use, instead of (14),

$$
y_{j}=\sum_{x} x p\left(y_{j} \mid x\right)
$$

where $p\left(y_{j} \mid x\right)$ is still given by (5).

The practical implementation of the algorithm at fixed temperature consists of alternate updating of eqns. (17) and (5), until convergence is reached. The temperature is then lowered and iteration process is restarted from the solution found at the previous temperature. Let us finally observe explicitly that when the temperature decreases below the critical value $T^{3 \rightarrow 4}$ the number of jets remains fixed $(K=4)$. Since we go to very small temperatures, $T \simeq 10^{-3}$, this means that the term $-T H$ in (7) is negligible; therefore, the final clusterization corresponds in practice to a minimum of the cost function

$$
D=\sum_{k=1}^{4} \sum_{x \in J_{k}} d\left(x, y_{k}\right),
$$


where $x \in J_{k}$ means that the particle $x$ belong to the jet $J_{k}$ of code-vector $y_{k}$. $D$ assumes the form (18) because the probabilities $p\left(x, y_{j}\right)$ assume only the values 1 or 0 , depending on the assignment of the particle of 4-momentum $x$ to the cluster $J_{k}$ or to another jet.

\section{$5 \quad$ Results and discussions}

Given the data set, be it simulated or real, one has to adopt a reconstruction criterion; we will use the following one ${ }^{\text {? }}$. Among the three possible pairings of the four jets $J_{\alpha}:\left\{\left(J_{1}, J_{2}\right),\left(J_{3}, J_{4}\right)\right\},\left\{\left(J_{1}, J_{3}\right),\left(J_{2}, J_{4}\right)\right\},\left\{\left(J_{1}, J_{4}\right),\left(J_{2}, J_{3}\right)\right\}$, with invariant masses $\left(m_{2 k}, m_{2 k+1}\right)$ ( $k=1,2,3$ for the three pairings), one chooses the one with the minimum value of

$$
\left|m_{2 k}-m_{2 k+1}\right|
$$

This method tends to underestimate the $W$ mass, a defect that could be corrected by different methods [15]; this analysis is however of no interest at the moment. Our results are presented in fig. 2. We plot the distribution of the $W$-mass for the reconstructed data set obtained using the Durham algorithm (on the left) and the Deterministic Annealing algorithm (on the right). One can see that the two algorithms provide similar results, giving as average value of the $W$ mass: $m_{W}=79.08 \mathrm{GeV} / \mathrm{c}^{2}$ for the Durham method and $m_{W}=79.33 \mathrm{GeV} / \mathrm{c}^{2}$ for DA.

The study of the differences between the two algorithms can be made using a quantitative definition of the similarity between the two clustering procedures. We introduce the similarity parameter $S$ by the formula:

$$
S=\frac{\sum_{i=1}^{n} \sum_{j=i+1}^{n} \min \left\{E_{i}, E_{j}\right\} \delta_{\alpha_{i j}^{1} \alpha_{i j}^{2}}}{\sum_{i=1}^{n} \sum_{j=i+1}^{n} \min \left\{E_{i}, E_{j}\right\}}
$$

where $\delta_{\alpha_{i j}^{1} \alpha_{i j}^{2}}$ is the Kronecker delta and

$$
\alpha_{i j}^{1}=1
$$

\footnotetext{
${ }^{3}$ Other methods are discussed in 15$]$.
} 

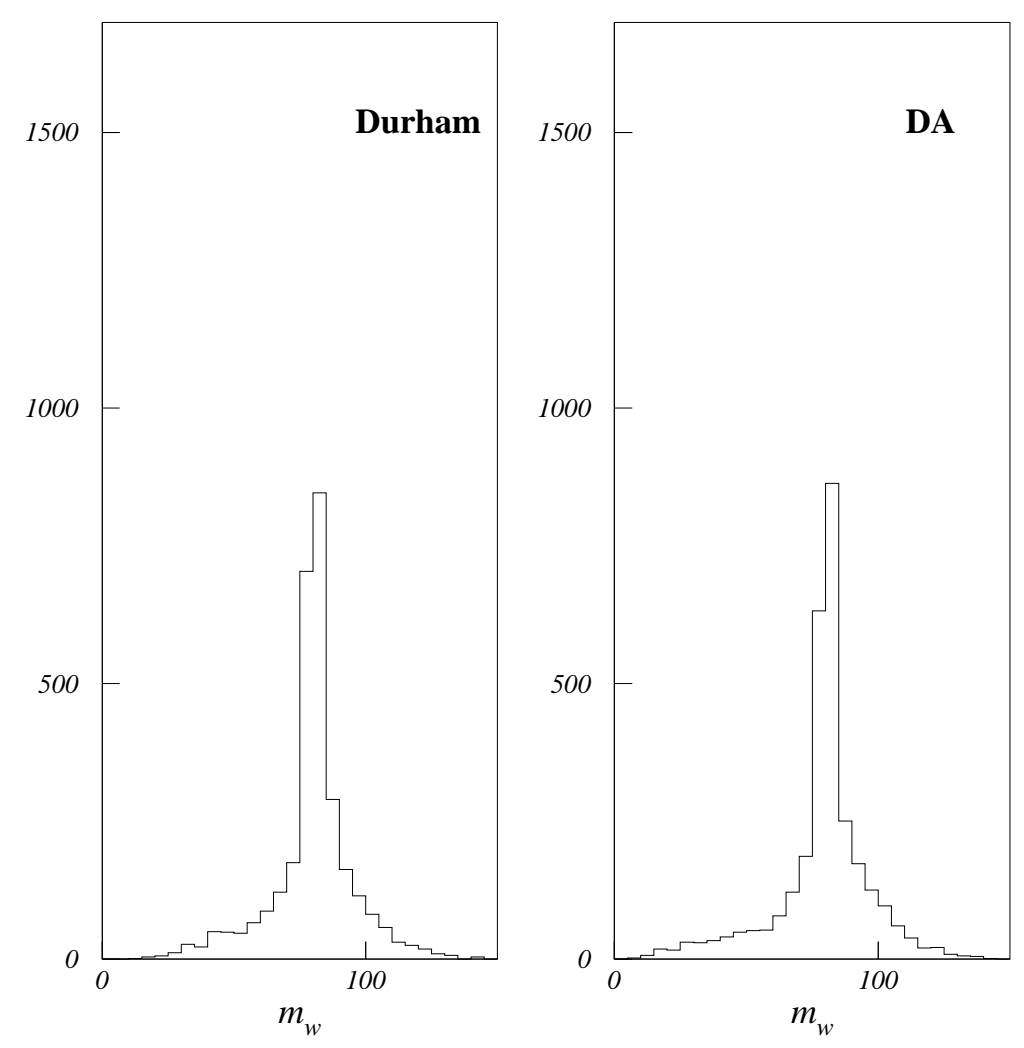

Figure 2: The $W$-mass (in $\mathrm{GeV} / \mathrm{c}^{2}$ ) distributions as reconstructed by the Durham jet algorithm (left) and Deterministic Annealing (right).

if the particles $i, j$ belong to the same cluster as defined by the Durham algorithm, while

$$
\alpha_{i j}^{1}=0
$$

otherwise; $\alpha_{i j}^{2}$ is defined in a similar way, but with the cluster identified by DA. It is clear that $S=1$ is equivalent to say that two particles are in the same cluster according to the Durham method if and only if they are in the same cluster also with DA; with $S=0$ this never happens. The factor $\min \left\{E_{i}, E_{j}\right\}$ gives lower weight to pairs with at least one low-energy particle. The histogram of the similarity $S$ is shown in Fig. 3: we find that a fraction of $87.8 \%$ of the events have $S>0.90$, i.e. to a large extent they are clustered identically by the two algorithms. It may be useful to observe that the percentage of events with $S>0.80$ is $99.0 \%$. Therefore we can 


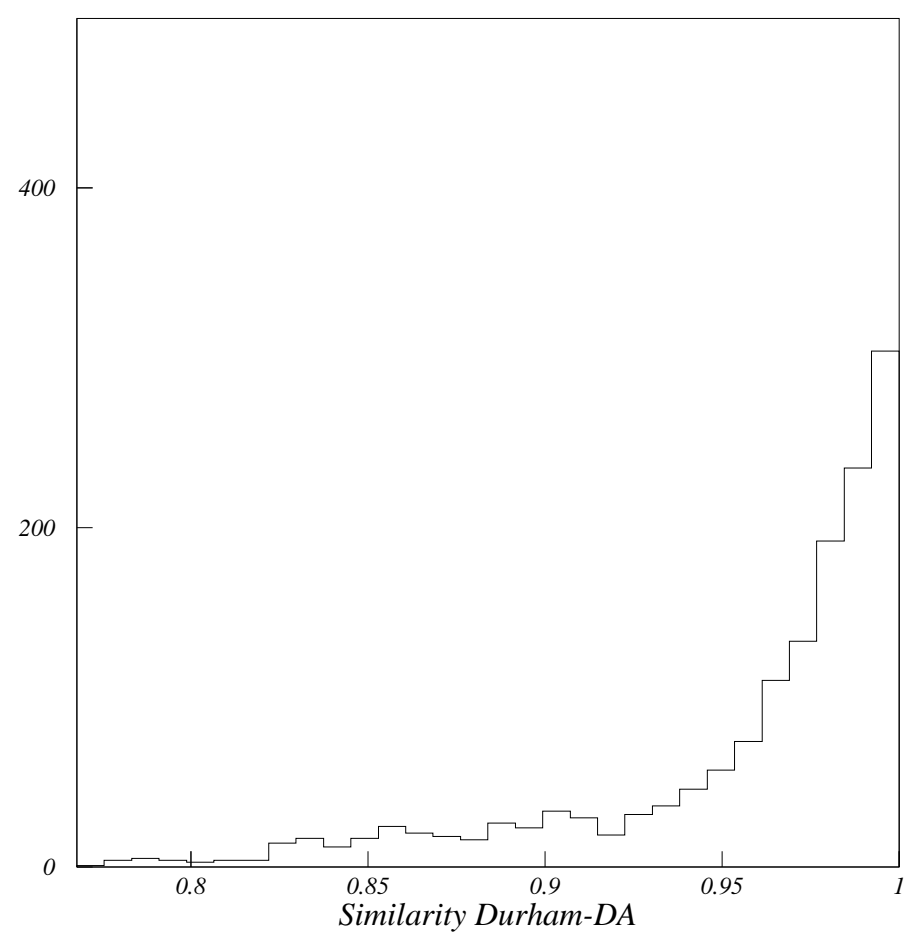

Figure 3: The distribution of the similarity $S$.

conclude that not only the two algorithms give similar results as far as the average $W$ mass is concerned, as we have already observed, but also that the composition of the jets provided by the two clustering procedures is identical in a very large fraction of the cases.

The consequence of this analysis is twofold; on one side we are insured that in a large majority of cases the Durham algorithm provides a minimum of the cost function (18); therefore we can say that, to a large extent, the Durham algorithm is not only a local, but also a global clusterization procedure.

On the other side, the results obtained so far show that the use of the DA algorithm might be seen as a practical alternative to the standard jet clusterization methods in the analyses involving huge computational tasks. This advantage may not be particularly important in the production of $W W$ pairs at the LEP energies, but could be significant at higher energies.

The reason why we expect a significant improvement at high multiplicities when using the DA algorithm lies in the computational complexity of 
DA which is of the order $N^{\alpha}$ ( $N=$ number of particles) with $\alpha \geq 1$, in comparison with $\alpha \geq 2$ for the Durham algorithm. As a matter of fact in the DA algorithm basically one performs one loop over the particles, see e.g. eq. (4) that contains a single loop over $x=1, \ldots, N$. On the other hand, in the case of the Durham algorithm there are two loops as shown by the discussion in section 2 (computation of $y_{i j}$ for $\left.i, j=1, \ldots, N\right)$. In both cases one expects values for $\alpha$ slightly larger than, respectively, $\alpha=1,2$; as a matter of fact in the DA case there are convergence criteria at fixed temperature to be satisfied; in the Durham case one performs other logical operations, in particular the reordering process, which also grows with $N$.

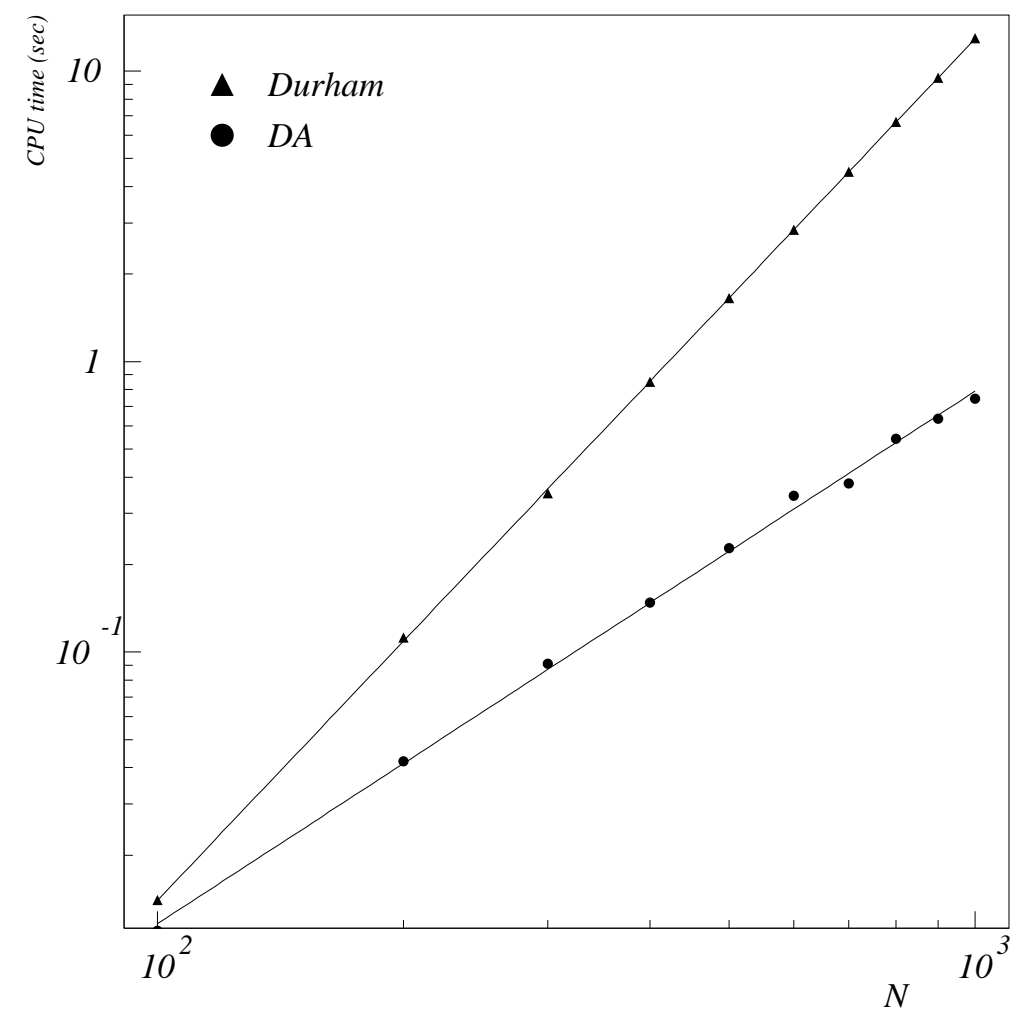

Figure 4: CPU time (in seconds), vs. particle multiplicity for the Deterministic Annealing algorithm (lower curve) and the Durham algorithm (upper curve).

We have performed the analysis of the computational complexity of the 
two algorithms for Monte Carlo generated $e^{+} e^{-}$events of increasing multiplicity. The results are reported in Fig. 1 where we compare the CPU time (in seconds) for the DA algorithm (lower curve) and for the Durham algorithm (upper curve) versus the multiplicity $N$.

A fit of the two curves of fig. 4 gives, for large $N$,

$$
\begin{array}{rll}
\text { Deterministic Annealing : } & t \propto N^{1.83} \\
\text { Durham algorithm }: & t \propto N^{2.97} .
\end{array}
$$

These results are obtained by a processor Pentium IV 1700.

\section{Conclusions}

We have compared the results found by the standard Durham algorithm with those obtained by a clustering algorithm based on the Deterministic Annealing procedure. The latter is a minimization algorithm, often used in clusterization problems, and has been adapted to the process studied here i.e. jet identification in particle production by high energy collisions. In particular we have addressed the study of the hadronic jets in $W W$ production by high energy $e^{+} e^{-}$scattering, but our results are rather general and should not depend on the specific reaction considered. They are as follows. First, we find that the two procedures give basically the same output as far as the clusterization is concerned. This means that one can interpret the Durham algorithm not only as a local computational scheme, but also as a global algorithm, i.e. a scheme attempting a minimization of a prescribed cost function. Second, we find that the CPU time of both algorithms increases with the particle multiplicity, but the growth is much faster for the Durham jet clustering algorithm, which is a consequence of the higher computational complexity of the Durham scheme in comparison with Deterministic Annealing. This result might be significant for jet physics at future accelerators such as the Large Hadron Collider at CERN.

\section{Acknowledgements}

We thank G. Dissertori for most useful discussions.

\section{References}


[1] K. Rose, E. Gurewitz and G. C. Fox, Pattern Rec. Letters 11 (1990) 589.

[2] K. Rose, Proc. of the IEEE 86 (1998) 2211.

[3] W. Bartel et al. [JADE Collaboration], Z. Phys. C33 (1986) 23; S. Bethke et al. [JADE Collaboration], Phys. Lett. B213 (1988) 235.

[4] Yu. L. Dokshitzer, in Proc. Workshop on Jet Studies at LEP and Hera, Durham, 1990, J. Phys. G 17 (1991) 1572ff; S. Catani, Yu. L. Dokshitzer, M. Olsson, G. Turnock and B. R. Webber, Phys. Lett. B269 432; N. Brown, W.J. Stirling, Z. Phys. C53 (1992) 629.

[5] Yu. L. Dokshitzer, G. Leder, S. Moretti and B. Webber, JHEP 9708 (1997) 001 hep-ph/9707323.

[6] S. Moretti, L. Lonnblad and T. Sjostrand, JHEP 9808 (1998) 001 hep-ph/9804296.

[7] A. Ballestrero et al., Report of the QCD working group, hep$\mathrm{ph} / 0006259$.

[8] M. Seymour, Z. Phys. C 62 (1994) 127; S. Bentvelsen and I. Meyer, Eur. Phys. J. C4(1998) 623 [hep-ph/9803322].

[9] S. Kirkpatrick, C.D. Gelatt and M.P. Vecchi, Science 220(1983) 671; V.Černy, J. Optimization Theory and Applications 45 (1985) 41.

[10] N. Metropolis, A. W. Rosenbluth, M. N. Rosenbluth, A. H. Teller and E. Teller, J. Chem. Phys. 21 (1953) 1087.

[11] D.E. Groom et al., The European Physical Journal C15 (2000) 1 (http://pdg.lbl.gov/).

[12] See the web page of the LEP Electroweak Working Group, http://lepewwg.web.cern.ch/LEPEWWG/Welcome.htm.

[13] M. Skrzypek, S. Jadach, W. Placzek and Z. Was, Comp. Phys. Commun. 94 (1996) 216.

[14] T. Sjöstrand, Comp. Phys. Commun. 82 (1994) 74; T. Sjöstrand, L. Lönnblad and S. Mrenna, Pythia 6.2 Physics and Manual, hepph/0108264. 
[15] R. Paramatti, PhD thesis, Università di Roma La Sapienza, 2002. 